\title{
SOLVABLE FUNDAMENTAL GROUPS OF COMPACT 3-MANIFOLDS
}

\author{
BY \\ BENNY EVANS AND LOUISE MOSER
}

\begin{abstract}
A classification is given for groups which can occur as the fundamental group of some compact 3-manifold. In most cases we are able to determine the topological structure of a compact 3-manifold whose fundamental group is known to be solvable. Using the results obtained for solvable groups, we are able to extend some known results concerning nilpotent groups of closed 3-manifolds to the more general class of compact 3-manifolds. In the final section it is shown that each nonfinitely generated abelian group which occurs as a subgroup of the fundamental group of a 3-manifold is a subgroup of the additive group of rationals.
\end{abstract}

(1) Introduction. This paper is primarily concerned with the classification of those solvable groups which can occur as the fundamental group of a compact 3-manifold. We also consider the problem of determining the structure of a compact 3-manifold whose fundamental group is known to be solvable. Our results are complete except in the category of almost sufficiently large 3-manifolds and the category of 3-manifolds whose nontrivial second homotopy group is generated by projective plane boundary components.

If $M$ is a compact, sufficiently large 3-manifold with trivial second homotopy group, and if $\pi_{1}(M)$ is solvable, then $\pi_{1}(M)$ appears in the following list of groups:

(1) $Z, Z \oplus Z$, or $\mathscr{K}$, the fundamental group of the Klein bottle,

(2) an extension $1 \rightarrow A \rightarrow \pi_{1}(M) \rightarrow Z \rightarrow 1$ where $A$ is either $Z \oplus Z$ or $\mathscr{K}$,

(3) a free product of two copies of $\mathscr{K}$ amalgamated along certain subgroups isomorphic with $Z \oplus Z$. These groups may be presented by $\left(a, b, x, y \mid b a b^{-1}=a^{-1}\right.$, $\left.y x y^{-1}=x^{-1}, a=x^{p} y^{2 q}, b^{2}=x^{r} y^{2 s}\right)$ where $p, q, r, s$ are integers such that $p s-r q= \pm 1$.

Further, the above list is complete. That is, for each group $G$ listed above, there is a compact sufficiently large 3-manifold $M$ with $\pi_{2}(M)=0$, and $\pi_{1}(M) \approx G$.

If the restrictions that $M$ be sufficiently large and that $\pi_{2}(M)=0$ are dropped, then further groups must be added to the list. Such groups are discussed in detail in $\S \S 3,4,5$, and 6 .

C. Thomas [16] has listed those nilpotent groups which can act as the fundamental group of a closed 3-manifold. Making use of the information we gain

Received by the editors February 2, 1971.

AMS 1970 subject classifications. Primary 57A10, 55A05; Secondary 20E15, $20 \mathrm{~K} 45$.

Key words and phrases. Solvable group, nilpotent group, sufficiently large 3-manifold, incompressible surface, Seifert fiber space, generalized free product, irreducible 3-manifold, nonfinitely generated abelian group. 
concerning solvable groups, we are able to simplify much of Thomas' work, and to extend his results to the bounded case. The only group which must be added to Thomas' list is $Z \oplus Z$.

In a remarkable paper of D. Epstein [1] those finitely generated abelian groups which occur as subgroups of the fundamental group of a 3-manifold are classified. We complete Epstein's list by proving that each nonfinitely generated abelian group which is a subgroup of the fundamental group of a 3-manifold is also a subgroup of the additive group of rationals.

(2) Definitions and preliminaries. If $g_{1}$ and $g_{2}$ are elements of the group $G$, we use the notation $\left[g_{1}, g_{2}\right]$ to denote the element $g_{1}^{-1} g_{2}^{-1} g_{1} g_{2}$ of $G$. If $H$ and $K$ are subgroups of the group $G$, we use the notation $[H, K]$ to denote the subgroup of $G$ generated by all elements $[h, k]$ of $G$, where $h \in H$ and $k \in K$. For a group $G$, we define the nth term $G^{(n)}$ of the commutator series of $G$, and the nth term $G_{(n)}$ of the lower central series of $G$ as follows: $G_{(0)}=G^{(0)}=G, G^{(n+1)}=\left[G^{(n)}, G^{(n)}\right]$, and $G_{(n+1)}=\left[G_{(n)}, G\right]$. A group $G$ is said to be solvable if there is an integer $n$ such that $G^{(n)}$ is the trivial group. A group $G$ is said to be nilpotent if there is an integer $n$ such that $G_{(n)}$ is the trivial group. Each nilpotent group is solvable. Also, each nilpotent group has nontrivial center. Each subgroup and each factor group of a nilpotent group is again nilpotent. Likewise, each subgroup and each factor group of a solvable group is a solvable group. Also each extension of a solvable group by a solvable group is again a solvable group.

We use the notation $Z_{p}$ to denote the cyclic group of order $p$ and $Z$ shall denote the infinite cyclic group.

Free groups of rank greater than one and fundamental groups of closed 2manifolds of Euler characteristic less than zero are not solvable. The fundamental group of the Klein bottle $\mathscr{K}$ is solvable. But $\mathscr{K}$ is not nilpotent since it has a centerless group $Z_{2} * Z_{2}$ as a factor group.

A group $G$ is said to be polycyclic if $G$ has a sequence of subgroups $G=G_{0} \supset G_{1}$ $\supset \ldots \supset G_{k}=1$ such that for each $i, G_{i+1}$ is a normal subgroup of $G_{i}$ and $G_{i} / G_{i+1}$ is a cyclic group. A polycyclic group $G$ is said to be poly-infinite-cyclic if the series above can be chosen so that $G_{i} / G_{i+1}$ is infinite cyclic for each $i, 0 \leqq i \leqq n-1$. Each subgroup and each factor group of a polycyclic group is polycyclic. Also each extension of a polycyclic group by a polycyclic group is again polycyclic. Each subgroup of a polycyclic group is finitely generated. Finally we remark that each finitely generated, torsion free, nilpotent group is poly-infinite-cyclic.

We refer the reader to the books by Marshall Hall [3] and Magnus, Karass, and Solitar [9] for the basic notions of group theory that we shall use here.

We shall use the notation $S^{n}$ to denote the standard $n$-dimensional sphere, and $P^{n}$ shall denote $n$-dimensional real projective space.

In this paper we assume that all spaces and maps are in the piecewise linear category. Also all subspaces are taken to be piecewise linear subspaces. If $A$ is a 
submanifold of the manifold $X$, we use the notation $\mathscr{U}(A, X)$ to denote a regular neighborhood of $A$ in $X$.

A 3-manifold $M$ is said to be irreducible if each 2-sphere in $M$ bounds a 3-cell in $M$. If $M$ is an irreducible 3-manifold and if in addition $M$ admits no two-sided embeddings of $P^{2}$, then we say that $M$ is $P^{2}$-irreducible.

A compact 3-manifold that is homotopy equivalent to a standard 3-simplex is a homotopy 3-cell. Of course it is not known if a homotopy 3-cell is necessarily homeomorphic with a standard 3-simplex.

Compact 3-manifolds $M_{1}, M_{2}$ are said to be simply equivalent if $M_{2}$ can be obtained from $M_{1}$ by either removing the interior of a homotopy 3-cell from the interior of $M_{1}$ or by sewing a homotopy 3-cell onto a 2-sphere boundary component of $M_{1}$. Compact 3-manifolds $M, M^{\prime}$ are said to be equivalent if there is a finite sequence of compact 3-manifolds $M=M_{0}, M_{1}, \ldots, M_{k}=M^{\prime}$ such that $M_{i}$ is simply equivalent to $M_{i+1}$ for each $i, 0 \leqq i<k$. van Kampen's theorem [10] assures us that equivalent 3-manifolds have isomorphic fundamental groups.

If $M$ is a compact 3-manifold, we have by Kneser's theorem [7] that $M$ is equivalent to a compact 3-manifold $\hat{M}$ such that $\hat{M}$ contains no 2 -sphere boundary components, and each 3-cell in $\hat{M}$ is homeomorphic with a standard 3-simplex. We shall find it convenient to adopt the convention suggested by the above remarks. That is, for each compact 3-manifold $M$ we shall use the notation $\hat{M}$ to denote a compact 3-manifold such that $\hat{M}$ is equivalent to $M, \hat{M}$ has no 2 -sphere boundary components, and each homotopy 3 -cell in $\hat{M}$ is homeomorphic with a standard 3-simplex.

If $X$ is a manifold we use bd $X$ to denote the boundary of $X$ and int $X$ to denote the interior of $X$.

A compact 2-manifold $F$ embedded in a 3-manifold $M$ is properly embedded in $M$ if $F \cap$ bd $M=$ bd $F$. A compact 2-manifold properly embedded in a 3-manifold $M$ is compressible in $M$ if there exists a disk $D$ in $M$ such that $D \cap F=$ bd $D$ and bd $D$ is not contractible in $F$. If $F$ is a compact 2-manifold that is properly embedded in a 3-manifold $M$ and if $F$ is not compressible in $M$, then $F$ is said to be incompressible in $M$.

Suppose $F$ is a compact, orientable 2-manifold with nonempty boundary. Suppose further that $F$ is properly embedded in a 3-manifold $M$. If an orientation is assigned to $F$, then an orientation is induced on bd $F$. We may then consider bd $F$ with its induced orientation as an element [bd $F$ ] of $H_{1}(\mathrm{bd} M)$. Since there are exactly two distinct orientations on $F$, we see that the homology class [bd $F$ ] is well defined up to a sign. If $F$ has no boundary, we take [bd $F$ ] to denote the trivial element of $H_{1}($ bd $M)$.

A compact 3-manifold $M$ is sufficiently large if $M$ contains a two-sided incompressible 2-manifold other than a disk or a 2-sphere.

F. Waldhausen [17] has shown that each compact, sufficiently large, orientable, irreducible 3-manifold has a hierarchy. That is to say that there is a sequence of 
compact 3-manifolds $M=M_{0} \supset M_{1} \supset \ldots \supset M_{k}$ and a sequence of compact 2manifolds $F_{0}, F_{1}, \ldots, F_{k-1}$ such that $F_{i}$ is a 2-sided, incompressible 2-manifold properly embedded in $M_{i}, M_{i+1}=\operatorname{cl}\left(M_{i}-\mathscr{U}\left(F_{\imath}, M_{i}\right)\right)$, and $M_{k}$ is a collection of 3-cells. Furthermore, the hierarchy can be chosen so that [bd $\left.F_{i}\right]$ is not trivial in $H_{1}\left(\right.$ bd $\left.M_{i}\right)$ for each $i(0<i \leqq k-1)$.

We shall use $I$ to denote the unit interval $[0,1]$.

Let $A$ and $B$ denote topological spaces. If $X$ is a locally trivial bundle with base $A$ and fiber $B$, then we shall refer to $X$ as a $B$ bundle over the topological space $A$. If $F$ is a closed 2-manifold and if $M$ is an $I$ bundle over $F$, then we say that $F$ is a trivial I bundle over $F$ if $M$ is homeomorphic with $F \times I$; otherwise, we say that $M$ is a twisted I bundle over $F$.

(3) Finite groups. By Corollary 8.7 of [1], each finite group which occurs as the fundamental group of a compact 3-manifold also occurs as the fundamental group of a closed, orientable 3-manifold. J. Milnor [11] has listed those finite groups which can occur as the fundamental group of a closed, orientable 3manifold. The theorems of this section are proved by checking each group in the list for the appropriate property.

THEOREM 3.1. Let $M$ be a compact 3-manifold such that $\pi_{1}(M)$ is finite. Then $\pi_{1}(M)$ is solvable unless $\pi_{1}(M)$ is isomorphic with the binary dodecahedral group $P_{120}$ or the direct sum of $P_{120}$ with a cyclic group of order relatively prime to 120 .

THEOREM 3.2. Let $M$ be a compact 3-manifold such that $\pi_{1}(M)$ is a finite nilpotent group. Then $\pi_{1}(M)$ is a finite cyclic group, a generalized quaternion group $Q\left(2^{k}\right)$, or the direct sum of $Q\left(2^{k}\right)$ with a cyclic group of odd order.

(4) The case $\pi_{2}(\hat{M})=0$ and $\pi_{1}(M)$ infinite. We shall make extensive use of the following lemma which appears as a problem in $\$ 4.1$ of [9]. The proof is omitted.

Lemma 4.1. If $A$ and $B$ are nontrivial groups, then $A * B$ is solvable if and only if $A \approx Z_{2} \approx B$.

THEOREM 4.2. Let $M$ be a compact 3-manifold such that $\pi_{2}(\hat{M})=0$. Suppose further that $\hat{M}$ has nonempty boundary. Then $\pi_{1}(M)$ is solvable if and only if $M$ is equivalent to a 3-manifold from the following list:

(i) a disk bundle over $S^{1}$;

(ii) an I bundle over the torus;

(iii) an I bundle over the Klein bottle.

We shall require two lemmas before beginning the proof of Theorem 4.2.

Lemma 4.3. Let $N$ be a compact $P^{2}$-irreducible 3-manifold with nonempty compressible boundary. Then $\pi_{1}(N)$ is solvable if and only if $N$ is homeomorphic with a disk bundle over $S^{1}$. 
Proof. Let $D$ be a disk properly embedded in $N$ such that bd $D$ is not contractible in bd $N$. Suppose $D$ separates $N$ into two components $N_{1}$ and $N_{2}$. As a consequence of the projective plane theorem [1], $N, N_{1}$, and $N_{2}$ are all aspherical 3-manifolds. Thus in particular Lemma 8.4 of [1] assures us that $\pi_{1}\left(N_{1}\right)$ contains no elements of finite order. By van Kampen's theorem $\pi_{1}(N) \approx \pi_{1}\left(N_{1}\right) * \pi_{1}\left(N_{2}\right)$. Observe that since bd $D$ is not contractible in bd $N$, it follows that neither $N_{1}$ nor $N_{2}$ is simply connected. Hence by Lemma $4.1, \pi_{1}\left(N_{1}\right) \approx Z_{2} \approx \pi_{1}\left(N_{2}\right)$. This is contrary to the statement above that $\pi_{1}\left(N_{1}\right)$ contains no elements of finite order. Hence $D$ does not separate $N$. Let $N^{*}=\operatorname{cl}(N-\mathscr{U}(D, N))$. By van Kampen's theorem $\pi_{1}(N) \approx \pi_{1}\left(N^{*}\right) * Z$. It follows that $N^{*}$ is simply connected. Since $N$ is irreducible it follows that $N^{*}$ is a 3-cell. The lemma follows.

LEMMA 4.4. Let $N$ be a compact, orientable, irreducible 3-manifold with nonempty, incompressible boundary. Then $\pi_{1}(N)$ is solvable if and only if $N$ is either $S^{1} \times S^{1} \times I$ or the twisted I bundle over the Klein bottle.

Proof. Let $N=N_{0} \supset N_{1} \supset \cdots \supset N_{k}$ be a hierarchy for $N$ where

$$
N_{\imath+1}=\operatorname{cl}\left(N_{i}-\mathscr{U}\left(F_{i}, N_{i}\right)\right), \quad 0 \leqq i<k .
$$

Assume further that the homology class [bd $F_{i}$ ] is not trivial in $H_{1}\left(\mathrm{bd} N_{i}\right)$ for each $i, 1 \leqq i<k$. For each $i$, bd $N_{i}$ is not empty. Thus the condition that [bd $F_{i}$ ] is not trivial in $H_{1}\left(\right.$ bd $\left.N_{i}\right)$ insures that each $N_{i+1}$ is connected, $0 \leqq i \leqq k-1$.

Since $F_{i}$ is incompressible and two-sided in $N_{\imath}$, it follows from the loop theorem [14] that the inclusion induced homomorphisms $i_{*}: \pi_{1}\left(F_{i}\right) \rightarrow \pi_{1}\left(N_{i}\right)$ and $j_{*}: \pi_{1}\left(N_{i+1}\right) \rightarrow \pi_{1}\left(N_{i}\right)$ are monomorphisms. Hence $\pi_{1}\left(F_{i}\right)$ and $\pi_{1}\left(N_{j}\right)$ are solvable groups for each $i, 0 \leqq i<k$, and for each $j, 0 \leqq j \leqq k$.

Observe that since $N_{k}$ is a 3-cell, $N_{k-1}$ must have compressible boundary. Let $r$ be the smallest integer such that bd $N_{r}$ is compressible in $N_{r}$. Then $N_{r}$ is a compact, orientable, irreducible 3-manifold with nonempty, compressible boundary. Furthermore, $\pi_{1}\left(N_{r}\right)$ is a solvable group. Hence by Lemma 4.3, $N_{r}$ is homeomorphic with $D \times S^{1}$ where $D$ denotes the standard 2-simplex.

Through the remainder of the proof, $j$ shall denote an arbitrary integer such that $0 \leqq j<r$. Observe that since $N_{j}$ is orientable and bd $N_{j}$ is incompressible, it follows that each component of bd $N_{j}$ is a torus. $F_{j}$ is a compact 2-manifold with nonempty boundary and solvable fundamental group. Since bd $N_{j}$ is incompressible, $F_{j}$ is not a disk. Also $F_{j}$ is two-sided and $N_{j}$ is orientable so that $F_{j}$ is not a möbius band. Hence $F_{j}$ is an annulus.

We identify $\mathscr{U}\left(F_{j}, N_{j}\right)$ with $F_{j} \times[0,1]$ in such a way that $F_{j}$ is identified with $F_{j} \times \frac{1}{2}$. We wish to observe that the two annuli $F_{j} \times 0$ and $F_{j} \times 1$ lie in the same boundary component of $N_{j+1}$. If the two boundary components of $F_{j}$ lie in different components of bd $N_{j}$, this is clear. If both boundary components of $F_{j}$ lie in the same boundary component of $N_{j}$, then bd $F_{j}$ bounds an annulus $A$ in bd $N_{j}$. Since [bd $F_{j}$ ] is not trivial in $H_{1}\left(\right.$ bd $\left.N_{j}\right)$, we see that $A \cup F_{j}$ is a Klein bottle. But 
$N_{j}$ is orientable. Hence $A \cup F_{j}$ is one-sided in $N_{j}$. It follows that $F_{j} \times 0$ and $F_{j} \times 1$ lie in the same boundary component of $N_{j+1}$.

From the above remarks, we observe that $N_{j}$ is obtained from $N_{j+1}$ as follows. Let $a$ and $b$ be a pair of disjoint simple closed curves in the same boundary component of $N_{j+1}$ such that neither $a$ nor $b$ is contractible in bd $N_{j+1}$. Let $h_{a}: F_{j} \times 0 \rightarrow \mathscr{U}\left(a\right.$, bd $\left.N_{j}\right)$ and $h_{b}: F_{j} \times 1 \rightarrow \mathscr{U}\left(b\right.$, bd $\left.N_{j+1}\right)$ be homeomorphisms. Then $N_{j}$ is obtained from $N_{j+1}$ by sewing $F_{j} \times[0,1]$ onto bd $N_{j}$ via the homeomorphisms $h_{a}$ and $h_{b}$.

As noted above, $N_{r}$ is homeomorphic with $D \times S^{1}$ where $D$ denotes the standard 2-simplex. Let $\beta=($ bd $D) \times 0$, let $x_{0}$ be a point on $\beta$, and put $\alpha=x_{0} \times S^{1}$. According to the remarks above, $N_{r-1}$ can be obtained from $N_{r}$ by sewing $F_{r} \times[0,1]$ onto bd $N_{r}$ by homeomorphisms that map the annuli $F_{j} \times 0, F_{j} \times 1$ onto regular neighborhoods of a pair of disjoint simple closed curves $a$ and $b$ in bd $N_{r}$. Since bd $N_{r}$ is a torus and $a$ and $b$ are not contractible in bd $N_{r}$, it follows that $a$ and $b$ are parallel. That is, up to a homotopy in bd $N_{r}, a=\alpha^{p} \beta^{q}$ and $b=\left(\alpha^{p} \beta^{q}\right)^{ \pm 1}$ where $p$ and $q$ are a pair of relatively prime integers. Then van Kampen's theorem yields the following presentation for $\pi_{1}\left(N_{r-1}\right):\left(x, y, z \mid y=x^{p}, z y z^{-1}=x^{ \pm p}\right)$. Let $G$ be the smallest normal subgroup of $\pi_{1}\left(N_{r-1}\right)$ containing the element $y$. Then $\pi_{1}\left(N_{r-1}\right) / G$ can be presented by $\left(x, z \mid x^{p}=1\right)$. Observe that this is also a presentation of the group $Z * Z p$. But $\pi_{1}\left(N_{r-1}\right)$ is solvable. Hence $p= \pm 1$. Thus we see that $N_{r-1}$ is an $I$ bundle over the torus or the Klein bottle. Since $N_{r-1}$ is orientable, it follows that $N_{r-1}$ is either $S^{1} \times S^{1} \times I$ or the twisted $I$ bundle over the Klein bottle.

We shall now show that $r=1$ and hence $N=N_{r-1}$. If $r \neq 1$ then $N_{r-2}$ can be obtained from $N_{r-1}$ by sewing $F_{r-2} \times[0,1]$ onto bd $\left(N_{r-1}\right)$ via homeomorphisms that map the annuli $F_{r-2} \times 0, F_{r-2} \times 1$ onto regular neighborhoods of a pair of disjoint, nontrivial curves in the same component of bd $N_{r-1}$.

If $N_{r-1}$ is homeomorphic with $S^{1} \times S^{1} \times I$, van Kampen's theorem yields the following presentation for $\pi_{1}\left(N_{r-2}\right):\left(x, y, z, w \mid[x, y]=1, \quad z=x^{p} y^{q}, \quad w z w^{-1}\right.$ $\left.=\left(x^{p} y^{q}\right)^{ \pm 1}\right)$ where $(p, q)$ is a pair of relatively prime integers. Let $G$ be the smallest normal subgroup of $\pi_{1}\left(N_{r-2}\right)$ containing the element $z$. Then $\pi_{1}\left(N_{r-2}\right) / G$ can be presented by $\left(x, y, w \mid[x, y]=1, x^{p} y^{q}=1\right)$. Let $H$ be the group with presentation $\left(x, y \mid[x, y]=1, x^{p} y^{q}=1\right)$. Observe that $H$ is not the trivial group for any values of $p$ and $q$. But $\pi_{1}\left(N_{r-2}\right) / G$ is isomorphic with the free product $Z * H$. Hence $\pi_{1}\left(N_{r-2}\right) / G$ is not a solvable group. It follows that if $N_{r-1}$ is $S^{1} \times S^{1} \times I$, then $r=1$ and $N=S^{1} \times S^{1} \times I$.

If $N_{r-1}$ is the twisted $I$ bundle over the Klein bottle, we obtain by van Kampen's theorem the following presentation for $\pi_{1}\left(N_{r-2}\right):\left(x, y, z, w \mid y x y^{-1}=x^{-1}\right.$, $\left.z=x^{p} y^{2 q}, w z w^{-1}=\left(x^{p} y^{2 q}\right)^{ \pm 1}\right)$. Let $H$ be the smallest normal subgroup of $\pi_{1}\left(N_{r-2}\right)$ containing the elements $x, y^{2}$ and $z$. Then $\pi_{1}\left(N_{r-2}\right) / H$ can be presented by $\left(y, w \mid y^{2}=1\right)$. But this is also a presentation for the nonsolvable group $Z * Z_{2}$. Hence $\pi_{1}\left(N_{r-2}\right)$ is not solvable. It follows that $r=1$, and $N$ is the twisted $I$ bundle over the Klein bottle. This completes the proof of Lemma 4.4. 
Proof of Theorem 4.2. Since $\pi_{2}(\hat{M})=0$ and each homotopy 3-cell in $\hat{M}$ is homeomorphic with the standard 3-simplex, it follows that $\hat{M}$ is $P^{2}$-irreducible. If bd $\hat{M}$ is compressible in $\hat{M}$, then by Lemma $4.3, \hat{M}$ is homeomorphic with a disk bundle over $S^{1}$. If $\hat{M}$ is orientable and bd $\hat{M}$ is incompressible, then, by Lemma $4.4, \hat{M}$ is an $I$ bundle over the torus or the Klein bottle. If $\hat{M}$ is nonorientable with incompressible boundary, we let $M^{*}$ denote the orientable double cover of $\hat{M}$. Then $M^{*}$ is equivalent to an $I$ bundle over either the torus or the Klein bottle. But $\hat{M}$ contains no two-sided projective planes. Thus by Theorem 1 of [5] we have that $\hat{M}$ is equivalent to an $I$ bundle over either the torus or the Klein bottle. This completes the proof of Theorem 4.2.

THEOREM 4.5. Let $M$ be a closed, sufficiently large 3-manifold with $\pi_{2}(M)=0$. Then $\pi_{1}(M)$ is solvable if and only if $M$ is equivalent to a 3-manifold from the following list:

(i) a torus bundle over $S^{1}$;

(ii) a Klein bottle bundle over $S^{1}$;

(iii) the union of two twisted I bundles over the Klein bottle sewn together along their boundaries.

Proof. Since $\pi_{2}(M)=0$, it follows that the associated manifold $\hat{M}$ is $P^{2}$ irreducible. Since $\hat{M}$ is sufficiently large, there is a two-sided incompressible 2manifold $F$ embedded in $M$. Furthermore, $F$ is not a 2-sphere. By the loop theorem [14], the homomorphism $i_{*}: \pi_{1}(F) \rightarrow \pi_{1}(\hat{M})$ induced by inclusion is a monomorphism. Hence $\pi_{1}(F)$ is a solvable group. Also $F$ is not a 2 -sphere, and since $F$ is two-sided, $F$ is not a projective plane. Hence $F$ is either a torus or a Klein bottle.

Case 1. F separates $M$. If $\hat{M}$ is nonorientable then $F$ can be chosen so that it does not separate $\hat{M}$. Thus in this case we may assume that $\hat{M}$ is orientable. Hence $F$ is homeomorphic with $S^{1} \times S^{1}$. The 2-manifold $F$ separates $\hat{M}$ into two components whose closures we denote by $M_{1}$ and $M_{2}$. Then $M_{1}$ and $M_{2}$ are compact, $P^{2}$-irreducible 3-manifolds with nonempty, incompressible boundary. Furthermore, the groups $\pi_{1}\left(M_{1}\right)$ and $\pi_{1}\left(M_{2}\right)$ are solvable. It follows then from Lemma 4.4 that $M_{1}$ and $M_{2}$ are each homeomorphic with an $I$ bundle over either the torus or the Klein bottle. Observe that $M_{1}$ and $M_{2}$ are orientable and that each manifold has connected boundary. It follows that $M_{1}$ and $M_{2}$ are both homeomorphic with a twisted $I$ bundle over the Klein bottle.

Case 2. $F$ does not separate $\hat{M}$. Let $M_{1}=\operatorname{cl}(\hat{M}-\mathscr{U}(F, \hat{M}))$. Then $M_{1}$ is a compact $P^{2}$-irreducible 3-manifold with nonempty, incompressible boundary. Furthermore, $\pi_{1}\left(M_{1}\right)$ is a solvable group. Thus $M_{1}$ is homeomorphic with an $I$ bundle over either the torus or the Klein bottle. Observe that bd $M_{1}$ is not connected. It follows that $M_{1}$ is homeomorphic with a product $G \times[0,1]$ where $G$ is either $S^{1} \times S^{1}$ or the Klein bottle. Hence $M$ is either a torus or Klein bottle bundle over $S^{1}$. 
It is straightforward to show that the fundamental groups of the manifolds (i) and (ii) are solvable.

The groups (iii) contain a normal subgroup $Z \oplus Z$ which is the fundamental group of the torus separating the $I$ bundles over the Klein bottles. The factor group obtained by moding out this normal subgroup is the solvable group $Z_{2} * Z_{2}$. Hence the groups (iii) are solvable. This completes the proof of Theorem 4.5 .

Let $M$ be a closed 3-manifold with $\pi_{2}(M)=0, \pi_{1}(M)$ solvable and $\beta_{1}(M)>0$. Then there exists an incompressible, two-sided 2-manifold embedded in $M$ which does not separate $M$. Hence we see from the proof of Theorem 4.5 that $M$ is equivalent to a torus or Klein bottle bundle over $S^{1}$.

COROLlaRY 4.6. The following is a complete list of all solvable groups $G$ which occur as the fundamental group of a compact, sufficiently large 3-manifold with trivial second homotopy group:

(i) $Z, Z \oplus Z$, or $\mathscr{K}$, the fundamental group of the Klein bottle;

(ii) an extension $1 \rightarrow A \rightarrow G \rightarrow Z \rightarrow 1$ where $A$ is either $Z \oplus Z$ or $\mathscr{K}$;

(iii) a free product of two copies of $\mathscr{K}$ amalgamated along certain subgroups isomorphic with $Z \oplus Z$. In this case $G$ has the presentation $\left(a, b, x, y \mid b a b^{-1}=a^{-1}\right.$, $\left.y x y^{-1}=x^{-1}, a=x^{p} y^{2 q}, b^{2}=x^{r} y^{2 s}\right)$ where $p, q, r, s$ are integers such that $p s-r q= \pm 1$.

Proof. If $G$ is the fundamental group of a compact, sufficiently large 3-manifold with trivial second homotopy group, then, by Theorems 4.2 and $4.5, G$ is among the groups listed above. Thus we need only show that each group $G$ in the list above actually occurs as the fundamental group of a compact, sufficiently large 3manifold $M_{G}$ with trivial second homotopy group. If $G$ is $Z, Z \oplus Z$, or $\mathscr{K}$, this is straightforward. Let $F$ denote either $S^{1} \times S^{1}$ or the Klein bottle, and suppose $G$ is an extension of $\pi_{1}(F)$ by $Z$. The generator of the group $Z$ acts on $\pi_{1}(F)$ by conjugation to induce an automorphism $\theta: \pi_{1}(F) \rightarrow \pi_{1}(F)$. By Baer's theorem [12] there exists a homeomorphism $h: F \rightarrow F$ such that the induced automorphism $h_{*}: \pi_{1}(F) \rightarrow \pi_{1}(F)$ is $\theta$. Let $M_{1}=F \times[0,1]$. Let $M_{G}$ be the identification space obtained from $M_{1}$ by identifying $(x, 0)$ with $(h(x), 1)$ for each $x$ in $F$. Then by van Kampen's theorem, $\pi_{1}\left(M_{G}\right) \approx G$.

Let $G$ be a group with presentation $\left(a, b, x, y \mid b a b^{-1}=a^{-1}, y x y^{-1}=x^{-1}\right.$, $\left.a=x^{p} y^{2 q}, b^{2}=x^{r} y^{2 s}\right), p s-r q= \pm 1$. We construct $M_{G}$ as follows. Let $M_{1}$ and $M_{2}$ denote twisted $I$ bundles over the Klein bottle. Then bd $M_{1}$ and bd $M_{2}$ are tori. Hence $\pi_{1}\left(\right.$ bd $\left.M_{1}\right) \approx Z \oplus Z \approx \pi_{1}\left(\right.$ bd $\left.M_{2}\right)$. Let $h$ : bd $M_{1} \rightarrow$ bd $M_{2}$ be a homeomorphism such that the induced isomorphism $h_{*}: Z \oplus Z \rightarrow Z \oplus Z$ has matrix $\left(\begin{array}{ll}p & q \\ r & s\end{array}\right)$. Let $M_{G}$ be the adjunction space $M_{1} U_{h} M_{2}$. By van Kampen's theorem $\pi_{1}\left(M_{G}\right) \approx G$.

LEMMA 4.7. Let $M$ be a compact 3-manifold with infinite, solvable fundamental group. Then $M$ has a finite sheeted, regular covering space $(\tilde{M}, p)$ such that $\beta_{1}(\tilde{M})>0$.

Proof. Let $G=\pi_{1}(M)$. Since $G$ is solvable, there exists an integer $k$ such that $G^{(k)}=1$. Since $G$ is infinite and $G^{(k)}=1$, there is an integer $i$ such that $G^{(i+1)}$ is of infinite index in $G^{(i)}$. Let $j$ be the smallest such integer. Let $(\tilde{M}, p)$ be the covering 
space of $M$ associated with the normal subgroup $G^{(j)}$ of $\pi_{1}(M)$. Then $(\tilde{M}, p)$ is a finite sheeted regular covering space of $M$. Furthermore $H_{1}(\tilde{M}) \approx G^{(j)} / G^{(j+1)}$ is infinite.

We use the notation $\mathrm{GL}_{n}(Z)$ to denote the multiplicative group of invertible, $n \times n$ matrices with entries from the ring of integers.

THEOREM 4.8. Let $M$ be a closed, nonsufficiently large 3-manifold with $\pi_{2}(M)=0$. Suppose further that $\pi_{1}(M)$ is an infinite, solvable group. Then $M$ has a finite sheeted, regular covering space $(\tilde{M}, p)$ such that $\tilde{M}$ is equivalent to a torus bundle over $S^{1}$. Furthermore, $(\tilde{M}, p)$ can be chosen so that the group of covering transformations of $(\tilde{M}, p)$ is a finite, solvable subgroup of $\mathrm{GL}_{n}(Z)$ with $n \leqq 3$.

Proof. Observe first of all that since $M$ is not sufficiently large, $M$ is an orientable 3-manifold. By Lemma $4.7, M$ has a finite sheeted, regular covering space $(\tilde{M}, p)$ such that $\beta_{1}(\tilde{M})>0$. Since $\tilde{M}$ is orientable, we see by the remarks following Theorem 4.5 that $\tilde{M}$ is equivalent to a torus bundle over $S^{1}$.

We have the exact sequence $1 \rightarrow \pi_{1}(\tilde{M}) \rightarrow \pi_{1}(M) \stackrel{f}{\rightarrow} Q \rightarrow 1$ where $Q$ denotes the finite group of covering transformations of $(\tilde{M}, p)$. We assume that among all finite sheeted, regular covering spaces $\left(N, p^{\prime}\right)$ of $M$ such that $\beta_{1}(N)>0,(\tilde{M}, p)$ is chosen so that the group $Q$ of covering transformations of $(\tilde{M}, p)$ has minimum order.

Since $\tilde{M}$ is compact, $H_{1}(\tilde{M})$ is a finitely generated, abelian group. Hence there exists a positive integer $\alpha$ such that $\alpha H_{1}(\tilde{M})=\left\{\alpha x \mid x \in H_{1}(\tilde{M})\right\}$ is torsion free. Furthermore, since $\tilde{M}$ is equivalent to a torus bundle over $S^{1}, \alpha H_{1}(\tilde{M})$ is isomorphic with $Z, Z \oplus Z$ or $Z \oplus Z \oplus Z$. Let $r$ be the rank of $\alpha H_{1}(\tilde{M})$. Then $r \leqq 3$.

Each covering transformation $q$ in $Q$ induces an automorphism

$$
q_{*}: H_{1}(\tilde{M}) \rightarrow H_{1}(\tilde{M}) \text {. }
$$

For $q \in Q$, let $\hat{q}$ denote the restriction of $q_{*}$ to $\alpha H_{1}(\tilde{M})$. Let $\psi: Q \rightarrow \mathrm{GL}_{r}(Z)$ be the homomorphism defined by $\psi(q)=\hat{q}$ for each $q$ in $Q$. We shall show that the assumption of minimum rank for $Q$ insures that $\psi$ is a monomorphism.

Let $Q^{\prime}$ denote the kernel of the homomorphism $\psi$. We have the following exact commutative diagram.

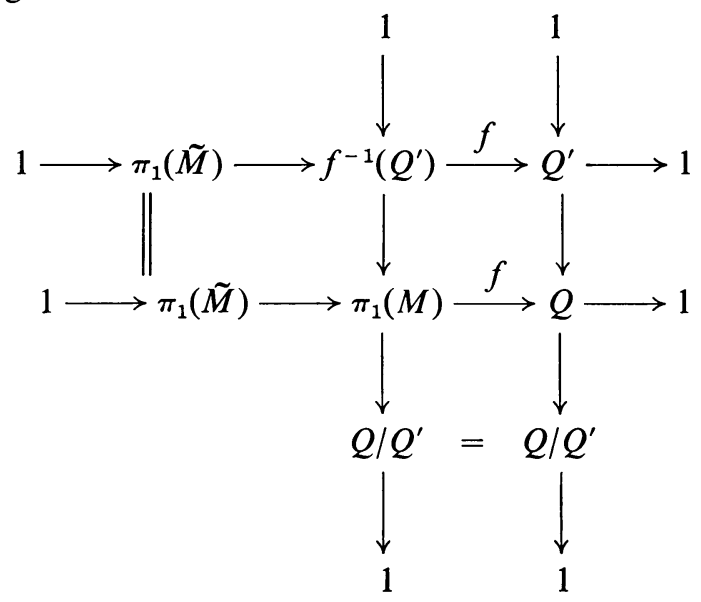


Let $\left(N, p^{\prime}\right)$ denote the regular covering space of $M$ associated with the normal subgroup $f^{-1}\left(Q^{\prime}\right)$ of $\pi_{1}(M)$. From the spectral sequence of a covering map [8] we obtain an exact sequence of terms of low degree. In particular we have the following exact sequence. $H_{2}\left(Q^{\prime}\right) \rightarrow H_{1}(\tilde{M}) \otimes_{Q^{\prime}} Z \rightarrow H_{1}(N) \rightarrow H_{1}\left(Q^{\prime}\right)$. We wish to show that $H_{1}(N)$ is infinite. Since $Q^{\prime}$ is finite, it follows that $H_{2}\left(Q^{\prime}\right)$ is also finite. Hence in order to show that $H_{1}(N)$ is infinite, we need only show that the tensor product $H_{1}(\tilde{M}) \otimes_{Q^{\prime}} Z$ is infinite. Let $K$ denote the subgroup of $H_{1}(\tilde{M})$ generated by all elements of the form $x-q_{*}(x)$ where $x \in H_{1}(\tilde{M})$ and $q \in Q^{\prime}$. Then $H_{1}(\tilde{M}) \otimes_{Q^{\prime}} Z$ is isomorphic with $H_{1}(\tilde{M}) / K$. Consider the subgroup $\left(\alpha H_{1}(\tilde{M})\right) \cap K$ of $H_{1}(\tilde{M})$. If $z \in\left(\alpha H_{1}(\tilde{M})\right) \cap K$, then there exist elements $x, y_{1}, \ldots, y_{k}$ of $H_{1}(\tilde{M})$, and there exist elements $q_{1}, \ldots, q_{k}$ of $Q^{\prime}$ such that $\alpha x=\sum_{i=1}^{k} y_{i}-q_{i_{*}}\left(y_{i}\right)$. Then $\alpha^{2} x=\sum_{i=1}^{k} \alpha y_{i}-q_{i_{*}}\left(\alpha y_{i}\right)=\sum_{i=1}^{k} \alpha y_{i}-\hat{q}_{i}\left(\alpha y_{i}\right)$. But $\hat{q}_{i}=\psi\left(q_{i}\right)$ is the identity map since $q_{i}$ is an element of the kernel of $\psi$. Hence $\alpha^{2} x=0$. But $\alpha H_{1}(\tilde{M})$ is torsion free. Thus $z=\alpha x=0$. It follows that $\left(\alpha H_{1}(\tilde{M})\right) \cap K=0$. Hence the restriction of the natural projection of $H_{1}(\tilde{M})$ onto $H_{1}(\tilde{M}) / K$ to $\alpha H_{1}(\tilde{M})$ is a monomorphism. Thus $H_{1}(\tilde{M}) \otimes_{Q^{\prime}} Z$ is infinite. It follows that $H_{1}(N)$ is also infinite.

Thus $\left(N, p^{\prime}\right)$ is a finite sheeted, regular covering space of $M$ such that $\beta_{1}(N)>0$. The group of covering transformations of $\left(N, p^{\prime}\right)$ is isomorphic with $Q / Q^{\prime}$. By the minimality assumption for $(\tilde{M}, p)$, the order of $Q$ is not greater than the order of $Q / Q^{\prime}$. It follows that $Q^{\prime}$ is the trivial group. Hence $\psi$ is a monomorphism and the proof of Theorem 4.8 is complete.

COROLlaRY 4.9. Let $G$ be the fundamental group of a compact, nonsufficiently large 3-manifold with trivial second homotopy group. Suppose further that $G$ is an infinite solvable group. Then $G$ is a nonsplit extension of the fundamental group of an orientable torus bundle over $S^{1}$ by a finite solvable subgroup of $\mathrm{GL}_{r}(Z), r \leqq 3$.

Looking closely at the proofs of this section, we have the following corollary.

COROLlaRY 4.10. Let $M$ be a compact sufficiently large 3-manifold with trivial second homotopy group. Then $\pi_{1}(M)$ is solvable if and only if $\pi_{1}(M)$ contains no free subgroup of rank two.

(5) The case $\pi_{2}(\hat{M}) \neq 0$. Let $G$ be a split extension of a group $H$ by a group $K$. Then each element $k$ of $K$ acts by conjugation on $H$ to induce an automorphism $\theta_{k}$ on $H$. We say that $K$ acts without fixed points on $H$ if for each $k$ in $K$ and each $h$ in $H, \theta_{k}(h)=h$ if and only if $k=1$ or $h=1$.

THEOREM 5.1. Suppose $M$ is a compact 3-manifold such that $\pi_{2}(\hat{M}) \neq 0$. If $\pi_{1}(M)$ is an infinite, solvable group, then precisely one of the following is true:

(i) $\pi_{1}(M) \approx Z, Z \oplus Z_{2}$, or $Z_{2} * Z_{2}$.

(ii) $\pi_{2}(\hat{M})$ is generated as a $\pi_{1}(\hat{M})$ module by the projective plane boundary components of $\hat{M}$, and $\pi_{1}(M)$ is a split extension of $G$ by $Z_{2}$ where $G$ is the fundamental group of a compact, orientable, irreducible, 3-manifold with solvable fundamental group. Furthermore, $Z_{2}$ acts without fixed points on $G$. 
If $M$ is a compact 3-manifold which falls into the second category of Theorem 5.1 , then $M$ can be obtained as follows. Let $N$ be a compact, orientable 3-manifold with $\pi_{2}(N)=0$ such that $\pi_{1}(N)$ is an infinite, solvable group. Let $f: N \rightarrow N$ be an involution whose fixed point set is a finite collection of points $x_{1}, \ldots, x_{k}$. Let $M_{1}$ be the orbit space cl $\left(N-\bigcup_{i=1}^{k} \mathscr{U}\left(x_{i}, N\right)\right) / f$. Then $M$ is obtained from $M_{1}$ by removing the interiors of a finite (possibly empty) collection of 3-cells.

Numerous examples of such 3-manifolds exist. For example we can construct an involution $\sigma$ on $N=S^{1} \times S^{1} \times[0,1]$ which fixes exactly four points as follows. Let $C$ denote the complex plane. The 2-manifold $S^{1} \times S^{1}$ is naturally embedded in $C \times C$ as the set of ordered pairs $\left(e^{2 \pi i \alpha}, e^{2 \pi i \beta}\right)$ such that $0 \leqq \alpha \leqq 1,0 \leqq \beta \leqq 1$. We define the involution $\tau: S^{1} \times S^{1} \rightarrow S^{1} \times S^{1}$ by

$$
\tau\left(e^{2 \pi i \alpha}, e^{2 \pi i \beta}\right)=\left(e^{2 \pi i(1-\alpha)}, e^{2 \pi i(1-\beta)}\right) .
$$

The involution $\tau$ fixes exactly four points; namely $x_{1}=(1,1), x_{2}=\left(1, e^{2 \pi i / 2}\right)$, $x_{3}=\left(e^{2 \pi i / 2}, 1\right), x_{4}=\left(e^{2 \pi i / 2}, e^{2 \pi i / 2}\right)$. Define $\sigma: N \rightarrow N$ by $\sigma(x, t)=(\tau(x), 1-t)$. Let $M$ be constructed from $N$ as described above. Then $\pi_{1}(M)$ has presentation $\left(x, y, t \mid[x, y]=1, t x t^{-1}=x^{-1}, t y t^{-1}=y^{-1}, t^{2}=1\right)$. Observe that since $Z_{2}$ acts without fixed points on $\pi_{1}(N) \approx Z \oplus Z$, it follows that $\pi_{1}(M)$ is the only split extension of $Z \oplus Z$ by $Z_{2}$ which occurs as the fundamental group of a compact 3-manifold.

Proof of Theorem 5.1. Since $\pi_{2}(\hat{M}) \neq 0$, we have by the projective plane theorem [1] that $\hat{M}$ contains either a noncontractible 2 -sphere or a two-sided projective plane.

Case 1. There exists a noncontractible 2-sphere $S$ in $\hat{M}$. If $S$ separates $\hat{M}$ into two components $M_{1}$ and $M_{2}$, then by van Kampen's theorem $\pi_{1}(\hat{M}) \approx \pi_{1}\left(M_{1}\right) * \pi_{1}\left(M_{2}\right)$. Since $S$ is not contractible in $\hat{M}$ and $\hat{M}$ has no 2-sphere boundary components, it follows that neither $M_{1}$ nor $M_{2}$ is simply connected. Hence $\pi_{1}\left(M_{1}\right) \approx Z_{2} \approx \pi_{1}\left(M_{2}\right)$. Thus $\pi_{1}(M) \approx \pi_{1}(\hat{M}) \approx Z_{2} * Z_{2}$.

If $s$ does not separate $\hat{M}$, then van Kampen's theorem allows us to write $\pi_{1}(\hat{M}) \approx Z * H$ where $H$ is the fundamental group of $\hat{M}-S$. Since $\pi_{1}(\hat{M})$ is solvable, it follows that $H$ is the trivial group. Hence $\pi_{1}(M) \approx \pi_{1}(\hat{M}) \approx Z$.

Case 2. Each 2-sphere in $\hat{M}$ is contractible in $\hat{M}$. Let $f_{1}, \ldots, f_{r}$ be maps of $S^{2}$ into the projective plane boundary components of $\hat{M}$ (if any exist) such that $f_{i}$ identifies antipodal points for each $i, 1 \leqq i \leqq r$. Let $\Lambda$ denote the $\pi_{1}(M)$-submodule of $\pi_{2}(\hat{M})$ generated by $f_{1}, \ldots, f_{r}$.

Case 2A. $\Lambda \neq \pi_{2}(M)$. By the projective plane theorem [1] there is a map $f: S^{2} \rightarrow \hat{M}$ such that $f \notin \Lambda$ and $f\left(S^{2}\right)$ is either a 2-sphere or a two-sided projective plane. Since each 2-sphere in $\hat{M}$ is contractible, it follows that $f\left(S^{2}\right)$ is a two-sided projective plane. Let $(\tilde{M}, P)$ denote the orientable double cover of $\hat{M}$, and let $\tilde{f}: S^{2} \rightarrow \tilde{M}$ be a lifting of $f$. Let $\tau: \tilde{M} \rightarrow \tilde{M}$ denote the nontrivial covering transformation of $(\tilde{M}, P)$. Suppose $f\left(S^{2}\right)$ does not separate $\hat{M}$. Let

$$
M_{1}=\operatorname{cl}\left(\hat{M}-\mathscr{U}\left(f\left(S^{2}\right), \hat{M}\right)\right)
$$


Suppose $\tilde{f}\left(S^{2}\right)$ separates $\tilde{M}$. Then since $\hat{M}-f\left(S^{2}\right)$ is connected, it follows that $\tau$ interchanges the components of $\tilde{M}-\tilde{f}\left(S^{2}\right)$. Let $S^{*}$ denote a 2-sphere in $\tilde{M}$ such that $S^{*}$ is parallel to $\tilde{f}\left(S^{2}\right)$. Then since $\tau\left(S^{*}\right) \cap S^{*}=\varnothing$, it follows that $\left.P\right|_{S^{*}}$ is an injection. Hence $P\left(S^{*}\right)$ is a noncontractible 2 -sphere in $\hat{M}$. Since each 2 -sphere in $\hat{M}$ is contractible, we have established a contradiction to the assumption that $\tilde{f}\left(S^{2}\right)$ separates $\tilde{M}$. Let $\tilde{M}_{1}=P^{-1}\left(M_{1}\right)$. Then $\pi_{1}(\tilde{M}) \approx Z * \pi_{1}\left(\tilde{M}_{1}\right)$. It follows that $\pi_{1}\left(\tilde{M}_{1}\right)=1$. Since $\tilde{M}_{1}$ is a connected, two-sheeted covering space of $M_{1}$, it follows that $\pi_{1}\left(M_{1}\right) \approx Z_{2}$. Then by Theorem 5.1 of [2], $M_{1}$ is homotopy equivalent with $P^{2} \times[0,1]$. It follows that $\pi_{1}(M) \approx \pi_{1}(\hat{M}) \approx Z \oplus Z_{2}$.

Suppose $f\left(S^{2}\right)$ separates $\hat{M}$ into two components whose closures we denote by $M_{1}$ and $M_{2}$. Let $\tilde{M}_{1}=P^{-1}\left(M_{1}\right)$, and let $\tilde{M}_{2}=P^{-1}\left(M_{2}\right)$. Since $\tilde{f}\left(S^{2}\right)$ is not in $P_{*}^{-1}(\Lambda)$, it follows that neither $\tilde{M}_{1}$ nor $\tilde{M}_{2}$ is simply connected. Thus since $\pi_{1}(\tilde{M}) \approx \pi_{1}\left(\tilde{M}_{1}\right) * \pi_{1}\left(\tilde{M}_{2}\right)$, we have that $\pi_{1}\left(\tilde{M}_{1}\right) \approx Z_{2} \approx \pi_{1}\left(\tilde{M}_{2}\right)$. But $P \mid \tilde{M}_{1}: \tilde{M}_{1} \rightarrow M_{1}$ is a two-sheeted covering map. Hence $\pi_{1}\left(M_{1}\right)$ is an extension of $Z_{2}$ by $Z_{2}$. This is not possible since as proved in [1], the only finite group which can occur as the fundamental group of a compact, nonorientable 3-manifold is $Z_{2}$.

Case 2B. $\Lambda=\pi_{2}(\tilde{M})$. Let $\tilde{f}_{1}, \ldots, \tilde{f}_{r}$ be liftings of the generators $f_{1}, \ldots, f_{r}$ of $\Lambda$ to the covering space $\tilde{M}$. The map $P_{*}: \pi_{2}(\tilde{M}) \rightarrow \pi_{2}(M)$ is an isomorphism of abelian groups. Thus since for each $i, 1 \leqq i \leqq r, P^{-1} f\left(S^{2}\right)$ is connected, it follows that $\pi_{2}(\tilde{M})$ is generated as a $\pi_{1}(\tilde{M})$ module by $\tilde{f}_{1}, \ldots, \tilde{f}_{r}$. Hence $\pi_{2}\left(\tilde{M}^{\wedge}\right)=0$. It follows that $\tilde{M}^{\wedge}$ is an irreducible, orientable, compact 3-manifold with infinite, solvable fundamental group.

Let $G=\pi_{1}(\tilde{M})$. Then $\pi_{1}(\hat{M})$ is an extension of $G$ by $Z_{2}$. The group $G$ is the fundamental group of an aspherical 3-manifold. Hence by Lemma 8.4 of [1], $G$ is torsion free. But $\hat{M}$ has at least one projective plane boundary component. Hence $\pi_{1}(\hat{M})$ contains a nontrivial element $t$ of order 2. It follows that the above extension is split by a homomorphism that maps the generator of $Z_{2}$ onto $t$.

The element $t$ acts on $G$ by conjugation to define an automorphism $\theta: G \rightarrow G$. Suppose there exists a nontrivial element $g$ in $G$ such that $\theta(g)=g$. Then $t g t^{-1}=g$. Since $G$ is torsion free, it follows that $t$ and $g$ generate a subgroup $H$ of $\pi_{1}(\hat{M})$ such that $H$ is isomorphic with $Z \oplus Z_{2}$. Then by Theorem 9.5 of [1], we can write $\hat{M}$ as a connected sum $\hat{M}=Q \# R$ where $\pi_{1}(Q) \approx Z \oplus Z_{2}$. By van Kampen's theorem, $\pi_{1}(\hat{M}) \approx \pi_{1}(Q) * \pi_{1}(R)$. Since $\pi_{1}(\hat{M})$ is solvable, it follows that $\pi_{1}(\hat{M})$ $\approx Z \oplus Z_{2}$. Then $\pi_{1}(\tilde{M}) \approx Z$. Since $\tilde{M}^{\wedge}$ is irreducible and orientable, it follows that $\tilde{M}^{\wedge}$ is homeomorphic with a product $D \times S^{1}$ where $D$ denotes the standard 2simplex. Thus $\hat{M}$ has a boundary component $T$ such that $T$ is isomorphic with either $S^{1} \times S^{1}$ or the Klein bottle. Since $\pi_{1}(\hat{M}) \approx Z \oplus Z_{2}$, we see that $T$ is compressible in $\hat{M}$. Let $D$ be a disk in $M$ such that $D \cap$ bd $\hat{M}=D \cap T=$ bd $D$, and bd $D$ is not contractible in $T$. If $D$ separated $\hat{M}$, van Kampen's theorem would allow us to write the fundamental group $Z \oplus Z_{2}$ of $\hat{M}$ as a nontrivial free product. Thus $D$ does not separate $\hat{M}$. Then by van Kampen's theorem $Z \oplus Z_{2} \approx \pi_{1}(\hat{M})$ $\approx Z * K$ where $K$ is the fundamental group of $\hat{M}-D$. This contradiction assures 
us that $Z_{2}$ acts on $G$ without fixed points. The proof of Theorem 5.1 is complete.

We are now able to prove a general theorem about solvable fundamental groups of compact 3-manifolds.

THEOREM 5.2. If $M$ is a compact 3-manifold then $\pi_{1}(M)$ is solvable if and only if $\pi_{1}(M)$ is polycyclic.

Proof. Let $M$ denote a compact 3-manifold with solvable fundamental group. A finite group $G$ is solvable if and only if $G$ is polycyclic. Thus we need only consider the case that $\pi_{1}(M)$ is infinite.

Case 1. $\pi_{2}(\hat{M})=0$. If $\hat{M}$ has nonempty boundary, then $\pi_{1}(M)$ is either $Z$, $Z \oplus Z$, or the fundamental group of the Klein bottle. All these groups are polycyclic. If $\hat{M}$ is closed, then we let $\tilde{M}$ denote the orientable double cover of $\hat{M}$ if $\hat{M}$ is nonorientable; otherwise, let $\tilde{M}=\hat{M}$. Then $\tilde{M}$ has a finite sheeted, regular covering space $M^{*}$ such that $M^{*}$ is equivalent to a torus bundle over $S^{1}$. Since $\pi_{1}\left(M^{*}\right)$ is polycyclic, it follows that $\pi_{1}(\tilde{M})$ is polycyclic. Hence $\pi_{1}(M) \approx \pi_{1}(\hat{M})$ is also polycyclic.

Case 2. $\pi_{2}(\hat{M}) \neq 0$. In this case $\pi_{1}(M)$ is either an extension of a polycyclic group by $Z_{2}$, or $\pi_{1}(M)$ is isomorphic with one of the polycyclic groups $Z, Z \oplus Z_{2}$ or $Z_{2} * Z_{2}$.

(6) Seifert fiber spaces. In this section we consider again the category of closed, $P^{2}$-irreducible 3-manifolds which are not sufficiently large but which have solvable, infinite fundamental groups. All such 3-manifolds known to the authors are Seifert fiber spaces. We therefore devote this section to the classification of those Seifert fiber spaces with solvable fundamental groups.

A Seifert fiber space $M$ is constructed as follows. Let $F$ be a closed 2-manifold and let $M_{1}$ denote a locally trivial $S^{1}$ bundle over $F$. Let $P: M_{1} \rightarrow F$ be the bundle projection. We shall refer to $F$ as the Seifert surface of $M$. Choose disjoint disks $D_{1}, \ldots, D_{k}$ in $F$ and set $M_{2}=\operatorname{cl}\left(M_{1}-\bigcup_{i=1}^{k} P^{-1}\left(D_{i}\right)\right)$. Note that $P^{-1}\left(D_{i}\right)$ is a solid torus for each $i, 1 \leqq i \leqq k$. We identify $P^{-1}\left(D_{i}\right)$ with $D_{i} \times S^{1}$. Put $q_{i}=\left(\right.$ bd $\left.D_{i}\right) \times 0$ and $h_{i}=x_{i} \times S^{1}$ where $x_{i}$ is a point on $q_{i}$. We complete $M$ by sewing solid tori $D_{i}^{\prime} \times S^{1}$ onto $q_{i} \times h_{i}$ in such a way that the curve (bd $\left.D_{i}^{\prime}\right) \times 0$ is sewn to the curve $q_{i}^{\alpha_{i}} h_{i}^{\beta_{i}}$ where $\left(\alpha_{i}, \beta_{i}\right)$ is a pair of relatively prime integers. If $y_{i}$ is the center of the disk $D_{i}$, we refer to the simple closed curve $P^{-1}\left(y_{i}\right)$ as a singular fiber of type $\left(\alpha_{i}, \beta_{i}\right)$. Observe that if $\alpha_{i}= \pm 1$ then $M$ can be refibered in such a way that $y_{i}$ no longer occurs as a singular fiber of $M$. Thus in the sequel, we lose no generality in assuming that $\left|\alpha_{i}\right|>1$ for each $i, 1 \leqq i \leqq k$.

If $F$ is orientable of genus $g$, van Kampen's theorem yields the following presentation for $\pi_{1}(M):\left(a_{i}, b_{i}, q_{j}, h \mid \prod_{i=1}^{g}\left[a_{l}, b_{i}\right]=h^{b} \prod_{i=1}^{k} q_{i},\left[h, q_{j}\right]=1, a_{i} h a_{i}^{-1}=h^{\varepsilon_{i}}\right.$, $\left.b_{i} h b_{i}^{-1}=h^{\lambda_{i}}, q_{j}^{\alpha} h^{\beta_{j}}=1\right), 1 \leqq i \leqq g, 1 \leqq j \leqq k, \varepsilon_{i}, \lambda_{i}= \pm 1$. In case $F$ is not orientable, $\pi_{1}(M)$ may be presented as $\left(c_{i}, q_{j}, h \mid \prod_{i=1}^{n} c_{i}^{2}=h^{b} \prod_{i=1}^{k} q_{i},\left[h, q_{j}\right]=1, c_{i} h c_{i}^{-1}=h^{\varepsilon_{i}}\right.$, $\left.q_{j}^{\alpha} h^{\beta_{j}}=1\right), 1 \leqq i \leqq n, 1 \leqq j \leqq k$. 
A Seifert fiber space $M$ with nonorientable Seifert surface $F$ and $k$ singular fibers of types $\left(\alpha_{1}, \beta_{1}\right), \ldots,\left(\alpha_{k}, \beta_{k}\right)$ has a double cover $\tilde{M}$, a Seifert fiber space with Seifert surface $\tilde{F}$, the orientable double cover of $F$, and $2 k$ singular fibers of types $\left(\alpha_{1}, \beta_{1}\right),\left(\alpha_{1}, \beta_{1}\right), \ldots,\left(\alpha_{k}, \beta_{k}\right),\left(\alpha_{k}, \beta_{k}\right)$. Hence we need only consider the case where $M$ has orientable Seifert surface. Furthermore, $\pi_{1}(M)$ is an extension of the normal, infinite cyclic subgroup generated by $h$.

Hence we have reduced the problem to classification of the planar discontinuous groups $G\left(g, k \mid \alpha_{1}, \ldots, \alpha_{k}\right)$ with presentation $\left(a_{i}, b_{i}, q_{j} \mid \prod_{i=1}^{g}\left[a_{i}, b_{i}\right]=\prod_{i=1}^{k} q_{i}, q_{j}^{\alpha}\right.$, $=1), 1 \leqq i \leqq g, 1 \leqq j \leqq k$.

LEMma 6.1. If $g \geqq 2$, or if $g=1$ and $k \geqq 1$, then $G\left(g, k \mid \alpha_{1}, \ldots, \alpha_{k}\right)$ is nonsolvable.

Proof. If $H$ is the smallest normal subgroup of $G\left(g, k \mid \alpha_{1}, \ldots, \alpha_{k}\right)$ generated by $q_{1}, \ldots, q_{k}$, then $G / H$ can be presented by $\left(a_{i}, b_{i} \mid \prod_{i=1}^{q}\left[a_{i}, b_{i}\right]=1\right), 1 \leqq i \leqq g$. This is also a presentation of the fundamental group of a closed 2-manifold of genus $g$. Thus if $g \geqq 2, G\left(g, k \mid \alpha_{1}, \ldots, \alpha_{k}\right)$ is not solvable.

If $g=1$ and $k \geqq 1$, then $G\left(g, k \mid \alpha_{1}, \ldots, \alpha_{k}\right)$ has a factor group $H$ with presentation

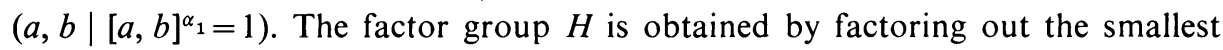
normal subgroup of $G\left(g, k \mid \alpha_{1}, \ldots, \alpha_{k}\right)$ containing the elements $q_{2}, \ldots, q_{k}$. Let $T$ denote a torus with the interior of a disk removed. Let $K$ denote the 2-complex obtained by sewing the boundary of a disk $D$ onto bd $T$ in such a way that bd $D$ wraps $\alpha_{1}$ times around bd $T$. Then by van Kampen's theorem $\pi_{1}(K) \approx H$. $K$ has a two-sheeted covering space $\tilde{K}$ obtained as follows. Let $\tilde{T}$ denote a torus with the interiors of two disks removed. $\tilde{K}$ is completed by sewing a disk onto each boundary component of $\tilde{T}$ so that each disk wraps $\alpha_{1}$ times around the boundary component onto which it is sewn. Then $\pi_{1}(\widetilde{K})$ is a subgroup of $\pi_{1}(K)$. By van Kampen's theorem, $\pi_{1}(\widetilde{K})$ has presentation $\left(a, b, t_{1}, t_{2} \mid[a, b]=t_{1} t_{2}, t_{1}^{\alpha}=t_{2}^{\alpha}=1\right) . \pi_{1}(\widetilde{K})$ has a factor group with presentation $\left(a, t_{1} \mid t_{1}^{\alpha}{ }_{1}=1\right)$. But this is also a presentation for the nonsolvable group $Z * Z_{\alpha_{1}}$. This completes the proof of Lemma 6.1.

If $A$ and $B$ are groups, and if $\phi: H \rightarrow K$ is an isomorphism between the subgroups $H$ of $A$ and $K$ of $B$, then we denote the free product of $A$ and $B$ amalgamated along the subgroups $H$ and $K$ by ${ }^{*}(A, B, H, K, \phi)$.

Lemma 6.2. Let $G={ }^{*}(A, B, H, K, \phi)$, where $A \neq H$ and $B \neq K$. Then $G$ is solvable if and only if $H$ is solvable and $H$ is of index two in $A$ and $K$ is of index two in $B$.

Proof. If $H$ is of index two in $A$ and $K$ is of index two in $B$, then observe that $G={ }^{*}(A, B, H, K, \phi)$ is an extension of $H$ by the solvable group $Z_{2} * Z_{2}$. Thus $G$ is solvable if and only if $H$ is.

The statement of the sufficiency of the lemma is a special case of a lemma of R. J. Gregorac [2]. Gregorac has shown that if $G=*(A, B, H, K, \phi)$ satisfies some nontrivial identity relation, then $H$ is of index two in $A$ and $K$ is of index two in $B$. Since each solvable group must satisfy some commutator identity (e.g. a group that is solvable of length two must satisfy the identity $[[X, Y],[Z, W]]=1)$, we may apply Gregorac's lemma above to obtain our result. 
THEOREM 6.3. $G\left(0, k \mid \alpha_{1}, \ldots, \alpha_{k}\right)$ is solvable in exactly the following cases:

(1) $k=4$ and $\alpha_{1}=\alpha_{2}=\alpha_{3}=\alpha_{4}=2$;

(2) $k=3$ and $\left(\alpha_{1}, \alpha_{2}, \alpha_{3}\right)=\left(2,2, \alpha_{3}\right),(2,3,3),(2,3,4),(2,3,6),(2,4,4)$, or $(3,3,3)$;

(3) $k \leqq 2$.

LEMmA A. If $G\left(0, k \mid \alpha_{1}, \ldots, \alpha_{k}\right)$ is solvable, then $k \leqq 4$. If $k=4$, then $\alpha_{1}=\alpha_{2}=\alpha_{3}$ $=\alpha_{4}=2$.

Proof. Since $k \geqq 4$, we may write $G=G\left(0,1 \mid \alpha_{1}, \ldots, \alpha_{k}\right)$ as $G={ }^{*}(A, B, H, K, \phi)$ where $A$ is the group presented by $\left(q_{1}, q_{2} \mid q_{i}^{\alpha_{i}}=1\right), B$ is the group presented by $\left(q_{3}, \ldots, q_{k} \mid q_{i}^{\alpha_{i}}=1\right), H$ is the subgroup of $A$ generated by $q_{1} q_{2}, K$ is the subgroup of $B$ generated by $q_{3} \cdots q_{k}$, and $\phi$ is the isomorphism of $H$ onto $K$ that maps $q_{1} q_{2}$ onto $\left(q_{3} \cdots q_{k}\right)^{-1}$. According to Lemma 6.2 if $G$ is to be solvable, then $H$ is of index two in $A$ and $K$ is of index two in $B$. Thus $A$ and $B$ are each extensions of $Z$ by $Z_{2}$. In particular both $A$ and $B$ are solvable groups. But $A$ and $B$ are free products of finite groups and hence by Lemma 4.1 can only be solvable if $k=4$ and $\alpha_{1}=\alpha_{2}$ $=\alpha_{3}=\alpha_{4}=2$.

LemmA B. If $\alpha_{1}, \alpha_{2}, \alpha_{3}$ are pairwise relatively prime, then $G\left(0,3 \mid \alpha_{1}, \alpha_{2}, \alpha_{3}\right)$ is nonsolvable.

Proof. If we abelianize $G\left(0,3 \mid \alpha_{1}, \alpha_{2}, \alpha_{3}\right)$, we have that $q_{1}^{\alpha_{1}}=1, q_{2}^{\alpha_{2}}=1, q_{1}^{\alpha_{3}} q_{2}^{\alpha_{3}}=1$. Then $q_{1}^{\alpha_{2} \alpha_{3}}=q_{1}^{\alpha_{2} \alpha_{3}} q_{2}^{\alpha_{2} \alpha_{3}}=\left(q_{1}^{\alpha_{3}} q_{2}^{\alpha_{3}}\right)^{\alpha_{2}}=1$. Since $\alpha_{1}$ and $\alpha_{2} \alpha_{3}$ are relatively prime, we have $q_{1}=1$. Similarly $q_{2}=q_{3}=1$. Hence $G\left(0,3 \mid \alpha_{1}, \alpha_{2}, \alpha_{3}\right)$ is in fact perfect.

We are now prepared to narrow our list of solvable groups $G\left(0, k \mid \alpha_{1}, \ldots, \alpha_{k}\right)$ to those given by Theorem 6.3. According to Lemma A, we need only consider the case $k=3$. Further, by Lemma B we need only consider groups $G\left(0,3 \mid \alpha_{1}, \alpha_{2}, \alpha_{3}\right)$ when there exists a prime $p$ that divides both $\alpha_{2}$ and $\alpha_{3}$. In such a case, we can construct a homomorphism of $G\left(0,3 \mid \alpha_{1}, \alpha_{2}, \alpha_{3}\right)$ onto $Z_{p}$ by mapping $q_{1}$ onto the identity element of $Z_{p}, q_{2}$ onto the generator of $Z_{p}$, and $q_{3}$ onto the inverse of the generator of $Z_{p}$. This map has kernel $K$ which may be presented by $\left(x_{i}, q_{2}^{\prime}, q_{3}^{\prime} \mid\right.$ $\left.\left(\prod_{i=0}^{p-1} x_{i}\right) q_{2}^{\prime} q_{3}^{\prime}=1, x_{i}^{\alpha}=1, q_{2}^{\prime} \alpha_{2}^{\prime}=1, q_{3}^{\prime} \alpha_{3}^{\prime}=1\right), 0 \leqq i<p, \alpha_{2}^{\prime}=\alpha_{2} / p, \alpha_{3}^{\prime}=\alpha_{3} / p$ under the map $x_{i} \mapsto q_{2}^{i} q_{1} q_{2}^{-i}, 0 \leqq i<p, q_{2}^{\prime} \mapsto q_{2}^{p}$, and $q_{3}^{\prime} \mapsto q_{3}^{p}$. Observe that $K$ is isomorphic with $G\left(0, p+2 \mid \alpha_{1}, \alpha_{1}, \ldots, \alpha_{1}, \alpha_{2} / p, \alpha_{3} / p\right)$.

(1) $G(0,3 \mid \alpha, p q, p r), q \neq 1, r \neq 1,(\alpha, p, q, r) \neq(2,2,2,2)$. This group has a nonsolvable subgroup $G(0, p+2 \mid \alpha, \alpha, \ldots, \alpha, q, r)$.

(2) $G(0,3 \mid \alpha, p, p), p \geqq 5$ or $p=3$ and $\alpha \geqq 4$. If $p \geqq 5$, then the subgroup $G(0, p \mid \alpha, \alpha, \ldots, \alpha)$ is not solvable. If $p=3$ and $\alpha \geqq 4$ then the subgroup $G(0,3 \mid \alpha, \alpha, \alpha)$ is not solvable by the above if $\alpha$ is a prime or by (1) if $\alpha=p^{\prime} q, p^{\prime} a$ prime and $q \neq 1$.

(3) $G(0,3 \mid \alpha, p, p r), r \neq 1, p \geqq 5$ has a subgroup $G(0, p+1 \mid \alpha, \alpha, \ldots, \alpha, r)$ which is not solvable.

(4) $G(0,3 \mid \alpha, 3,3 r), r \neq 1$ and $\alpha \neq 2$ or $r \neq 2$. The subgroup $G(0,4 \mid \alpha, \alpha, \alpha, r)$ is not solvable. 
(5) $G(0,3 \mid \alpha, 2,2 r), r \neq 1$ and $\alpha \geqq 5$ or $\alpha=4, r \geqq 3$, or $\alpha=3, r \geqq 4$. The subgroup $G(0,3 \mid \alpha, \alpha, r)$ is not solvable. If $\alpha \geqq 5$ this follows from (2) if $\alpha$ is prime and by (1) if $\alpha$ is composite. If $\alpha=4$ and $r \geqq 3$, this follows from (1). If $\alpha=3$ and $r \geqq 4$ this follows from (4).

The only groups remaining to be considered are those listed in Theorem 6.3. It is straightforward to show that each of these groups is solvable. We have the following theorem.

THEOREM 6.4. Let $M$ be a Seifert fiber space such that $\pi_{1}(M)$ is solvable. Then $M$ has Seifert surface $F$ either the torus, the Klein bottle, the projective plane, or the 2-sphere. Furthermore, if $F$ is the torus or the Klein bottle then $M$ has no singular fibers. If $F$ is the projective plane, then $M$ has no singular fibers, one singular fiber of arbitrary type, or two singular fibers of types $\left(2, \beta_{1}\right),\left(2, \beta_{2}\right)$. If $F$ is the 2-sphere then $M$ has zero, one, or two singular fibers of arbitrary types, three singular fibers of types $\left(2, \beta_{1}\right),\left(2, \beta_{2}\right),\left(\alpha_{3}, \beta_{3}\right) ;\left(2, \beta_{1}\right),\left(3, \beta_{2}\right),\left(3, \beta_{3}\right) ;\left(2, \beta_{1}\right),\left(3, \beta_{2}\right),\left(4, \beta_{3}\right) ;\left(2, \beta_{1}\right)$, $\left(3, \beta_{2}\right),\left(6, \beta_{3}\right) ;\left(2, \beta_{1}\right),\left(4, \beta_{2}\right),\left(4, \beta_{3}\right) ;\left(3, \beta_{1}\right),\left(3, \beta_{2}\right),\left(3, \beta_{3}\right)$; or four singular fibers of types $\left(2, \beta_{1}\right),\left(2, \beta_{2}\right),\left(2, \beta_{3}\right),\left(2, \beta_{4}\right)$.

In particular, we wish to call attention to the Seifert fiber spaces $M$ with Seifert surface a 2 -sphere with three singular fibers of types $\left(2, \beta_{1}\right),\left(3, \beta_{2}\right),\left(6, \beta_{3}\right) ;\left(2, \beta_{1}\right)$, $\left(4, \beta_{2}\right),\left(4, \beta_{3}\right)$; or $\left(3, \beta_{1}\right),\left(3, \beta_{2}\right),\left(3, \beta_{3}\right)$ with the additional restriction that $\beta_{1} \alpha_{2} \alpha_{3}+\alpha_{1} \beta_{2} \alpha_{3}+\alpha_{1} \alpha_{2} \beta_{3}+b \alpha_{1} \alpha_{2} \alpha_{3} \neq 0$. These manifolds have infinite, solvable fundamental groups. However, in [18] Waldhausen has shown that these manifolds are not sufficiently large. These manifolds constitute the complete collection of compact, nonsufficiently large, $P^{2}$-irreducible 3-manifolds with infinite, solvable fundamental groups known to the authors.

(7) Nilpotent groups. In [16] Thomas classifies those nilpotent groups which occur as the fundamental group of a closed 3-manifold. Here we provide a somewhat simpler proof of the results of Thomas and extend them to the bounded case.

If $M$ is a torus bundle over $S^{1}$, then we have an exact sequence $1 \rightarrow Z \oplus Z$ $\rightarrow \pi_{1}(M) \rightarrow Z \rightarrow 1$. The generator of the infinite cyclic quotient group induces an automorphism $\theta_{M}: Z \oplus Z \rightarrow Z \oplus Z$. The automorphism $\theta_{M}$ determines the fundamental group of $M$ up to isomorphism. Each torus bundle over $S^{1}$ is a $P^{2}$ irreducible, sufficiently large 3-manifold. Hence by a theorem of W. Heil [4], if $N$ is any other torus bundle over $S^{1}$, and if $\theta_{M}=\theta_{N}$, then $M$ is homeomorphic with $N$.

THEOREM 7.1. Let $M$ be a compact 3-manifold such that $\pi_{2}(\hat{M})=0$. Then $\pi_{1}(M)$ is an infinite, nilpotent group if and only if $M$ is equivalent to a manifold from the following list:

(i) a disk bundle over $S^{1}$;

(ii) an I bundle over $S^{1} \times S^{1}$;

(iii) a torus bundle $N$ over $S^{1}$ with $\theta_{N}$ of the form $\left(\begin{array}{ll}1 & Y \\ 0 & 1\end{array}\right), Y$ an integer. 
Proof. Since $\pi_{2}(\hat{M})=0$, it follows that $\hat{M}$ is $P^{2}$-irreducible. If $\hat{M}$ has nonempty boundary, then since $\pi_{1}(M)$ is solvable we may apply Theorem 4.2. Eliminating the nonnilpotent fundamental group of the Klein bottle from the list of Theorem 4.2, we have that $\hat{M}$ is equivalent to either a disk bundle over $S^{1}$ or an $I$ bundle over the torus.

If $\hat{M}$ is closed, we could again refer to the work in $\S 4$ on solvable groups; however, an easier approach is available. Since $\hat{M}$ is aspherical, it follows from Lemma 8.4 of [1] that $\pi_{1}(\hat{M})$ is torsion free. Hence $\pi_{1}(\hat{M})$ is a finitely generated, torsion free, nilpotent group. It follows that $\pi_{1}(\hat{M})$ is poly-infinite-cyclic. Thus there is a map $f$ of $\pi_{1}(\hat{M})$ onto the infinite cyclic group. Furthermore, since $\pi_{1}(\hat{M})$ is polycyclic, it follows that each subgroup of $\pi_{1}(\hat{M})$ is finitely generated. In particular the kernel of the map $f$ is finitely generated. It follows then from a theorem of Stallings [15] that $\hat{M}$ is homeomorphic with a closed 2-manifold bundle over $S^{1}$. Since $\pi_{1}(\hat{M})$ is nilpotent, $\hat{M}$ is a torus bundle over $S^{1}$.

We refer to Lemma 2 of Thomas' paper [16] for a proof that $\theta_{\hat{M}}$ is of the form $\left(\begin{array}{ll}1 & Y \\ 0 & 1\end{array}\right)$.

THEOREM 7.2. Let $M$ be a compact 3-manifold such that $\pi_{2}(\hat{M}) \neq 0$. Then $\pi_{1}(M)$ is an infinite, nilpotent group if and only if $\pi_{1}(M)$ is either $Z$ or $Z \oplus Z_{2}$.

Proof. If $\pi_{2}(\hat{M})$ is not generated as a $\pi_{1}(\hat{M})$ module by its projective plane boundary components, then by Theorem $5.1, \pi_{1}(M)$ is either $Z, Z \oplus Z_{2}$ or $Z_{2} * Z_{2}$. Since $Z_{2} * Z_{2}$ is centerless, it is not nilpotent.

If $\pi_{2}(\hat{M})$ is generated by the projective plane boundary components of $\hat{M}$, then by Theorem $5.1, \pi_{1}(M)$ is a split extension of a nontrivial group $G$ by $Z_{2}$. Furthermore, $Z_{2}$ acts without fixed points on $G$. Suppose the extension is split by a homomorphism that maps the generator of $Z_{2}$ onto an element $t$ of $\pi_{1}(M)$. Then each element of $\pi_{1}(M)$ can be written in the form $g t^{\varepsilon}$ where $g \in G$ and $\varepsilon$ is either 0 or 1 . Since $\pi_{1}(M)$ is nilpotent, there is a nontrivial element $g_{0} t^{\varepsilon}$ in the center of $\pi_{1}(M)$. Since $Z_{2}$ acts without fixed points on $G, \varepsilon \neq 0$. For otherwise $g_{0} t^{\varepsilon}$ does not commute with $t$. Hence $g_{0} t^{\varepsilon}=g_{0} t$. Then $t^{-1} g_{0} t=t^{-1}\left(g_{0} t\right)=\left(g_{0} t\right) t^{-1}=g_{0}$. Since $Z_{2}$ acts without fixed points on $G$, it follows that $g_{0}=1$. But then $g_{0} t^{\varepsilon}=t$ and $t$ does not commute with any nontrivial element of $G$. Hence $\pi_{1}(M)$ is a centerless group. This contradiction completes the proof of Theorem 7.2.

(8) Abelian groups. In [1] Epstein lists those finitely generated abelian groups which can occur as the fundamental group of a (possibly noncompact) 3-manifold. In this section we prove that if $M$ is a 3-manifold with nonfinitely generated, abelian fundamental group, then $\pi_{1}(M)$ is a subgroup of the additive group of rationals. The authors wish to thank William Jaco for considerable help in proving the theorems of this section.

An abelian group $G$ is said to be of local rank one if each finitely generated subgroup of $G$ is cyclic. The primary algebraic tool that we shall require is the following theorem which is proved in [6]. 
THEOREM 8.1. If $G$ is a torsion free abelian group of local rank one, then $G$ is a subgroup of the additive group of rationals.

LEMMA 8.2. If $M$ is a 3-manifold with nonfinitely generated abelian group then $\pi_{1}(M)$ is torsion free.

Proof. Suppose $\pi_{1}(M)$ contains a nontrivial element $x$ of order $k$, and let $H$ denote the subgroup $\pi_{1}(M)$ generated by $x$. Then $H$ is isomorphic with $Z_{k}$. If $k$ is not two, then Theorem 8.2 of [1] allows us to write $M$ as a connected sum $M=Q \# R$ where $\pi_{1}(R)$ is a nontrivial finite group. By van Kampen's theorem $\pi_{1}(M) \approx \pi_{1}(Q) * \pi_{1}(R)$. Then since $\pi_{1}(M)$ is abelian we have that $\pi_{1}(Q)=0$ and $\pi_{1}(M)=\pi_{1}(R)$. This of course is contrary to the assumption that $\pi_{1}(M)$ is not finitely generated.

Thus we have that each element of $\pi_{1}(M)$ is either of infinite order or has order 2. Let $y$ denote a nontrivial element of $\pi_{1}(M)$ other than the torsion element $x$ above, and let $K$ denote the subgroup of $\pi_{1}(M)$ generated by the elements $x$ and $y$. If $y$ were of finite order, then as noted above we would have $y^{2}=1$ and so $K$ would be isomorphic with $Z_{2} \oplus Z_{2}$. But Theorem 9.1 of [1] assures us that $Z_{2} \oplus Z_{2}$ is not a subgroup of the fundamental group of any 3-manifold. Thus $y$ must be of infinite order and so $K$ is isomorphic with $Z \oplus Z_{2}$. Then by Theorem 9.5 of [1] $M$ can be written as a connected sum $M=Q \# R$ with $\pi_{1}(R) \approx Z \oplus Z_{2}$. Since $\pi_{1}(M)$ is not a free product, we have $\pi_{1}(M) \approx Z \oplus Z_{2}$. This is contrary to the assumption that $\pi_{1}(M)$ is not finitely generated.

This completes the proof of Lemma 8.2.

We are now ready to prove the main theorem of this section.

THEOREM 8.3. If $M$ is a 3-manifold and if $\pi_{1}(M)$ is a nonfinitely generated abelian group then $\pi_{1}(M)$ is a subgroup of the additive group of rationals.

Proof. In view of Theorem 8.1 and Lemma 8.2, we need only prove that $\pi_{1}(M)$ is of local rank one. Furthermore, it suffices to prove the theorem in the case that $M$ is orientable. For if $M$ is nonorientable we consider the orientable double cover $\tilde{M}$ of $M$. Assuming the theorem known for orientable manifolds, we have the exact sequence $1 \rightarrow \pi_{1}(\tilde{M}) \rightarrow \pi_{1}(M) \rightarrow Z_{2} \rightarrow 1$ where $\pi_{1}(M)$ is torsion free and $\pi_{1}(\tilde{M})$ is of local rank one. It is then a simple exercise to show that $\pi_{1}(M)$ is also of local rank one.

Thus we need only establish that if $M$ is an orientable 3-manifold with nonfinitely generated, abelian fundamental group then $\pi_{1}(M)$ is of local rank one. The proof is by contradiction. Thus we assume that $M$ is an orientable 3-manifold with nonfinitely generated abelian fundamental group and $\pi_{1}(M)$ is not of local rank one. Then $\pi_{1}(M)$ contains a subgroup $H$ isomorphic with $Z \oplus Z$. Let $(N, P)$ denote the covering space of $M$ associated with the subgroup $H$ of $\pi_{1}(M)$ and let $C$ denote the group of covering transformations of $(N, P)$. 
Lemma A. There exists an embedding $f: S^{1} \times S^{1} \rightarrow N$ such that $f_{*}: \pi_{1}\left(S^{1} \times S^{1}\right)$ $\rightarrow \pi_{1}(N)$ is an isomorphism.

Proof. Let $g_{1}: S^{1} \times S^{1} \rightarrow N$ be a map such that $g_{1}: \pi_{1}\left(S^{1} \times S^{1}\right) \rightarrow \pi_{1}(N)$ is an isomorphism, and let $K_{1}$ denote a regular neighborhood of $g_{1}\left(S^{1} \times S^{1}\right)$ in $N$. If the boundary of $K_{1}$ is compressible in $N$, then there is a disk $D$ in $N$ such that $D \cap$ bd $K_{1}$ $=$ bd $D$ and bd $D$ is a nontrivial curve in bd $K_{1}$. If $D \cap$ int $K_{1}=\varnothing$, then we set $g_{2} \equiv g_{1}$, and $K_{2}=K_{1} \cup \mathscr{U}(D, N)$. Otherwise we have $D \subset K_{1}$. We change $g_{1}$ by a small isotopy if necessary so that $g_{1}$ is a general position map with respect to the disk $D$. Then $g_{1}^{-1}\left(g_{1}\left(S^{1} \times S^{1}\right) \cap D\right)$ consists of a collection of mutually exclusive simple closed curves on $S^{1} \times S^{1}$. If $\alpha$ is a component of $g_{1}^{-1}\left(g_{1}\left(S^{1} \times S^{1}\right) \cap D\right)$ then $g_{1}(\alpha) \subset D$ so that $g_{1}(\alpha)$ is contractible in $N$. Since $g_{1}$. is an isomorphism, it follows that $\alpha$ is contractible on $S^{1} \times S^{1}$. Thus each simple closed curve $\alpha$ in $g_{1}^{-1}\left(g_{1}\left(S^{1} \times S^{1}\right) \cap D\right)$ bounds a disk $D_{\alpha}$ on $S^{1} \times S^{1}$. Choose new base points if necessary so that the base point of $S^{1} \times S^{1}$ does not lie on any disk $D_{\alpha}$ for $\alpha$ a component of $g_{1}^{-1}\left(g_{1}\left(S^{1} \times S^{1}\right) \cap D\right)$. $g_{1}$. remains an isomorphism. Now let $\alpha$ denote a fixed component of $g_{1}^{-1}\left(g_{1}\left(S^{1} \times S^{1}\right) \cap D\right)$. Define $g_{2}: S^{1} \times S^{1} \rightarrow N$ as follows. We set $\left.g_{2}\right|_{\mathrm{cl}\left(S^{1} \times S^{1}-D_{\alpha}\right)}=\left.g_{1}\right|_{\mathrm{cl}\left(S^{1} \times S^{1}-D_{\alpha}\right)}$, and $\left.g_{2}\right|_{D_{\alpha}}$ is a contraction of $g_{2}(\alpha)$ in $D$. We then change $g_{2}$ by a small homotopy so that $g_{2}\left(D_{\alpha}\right)$ lies on the proper side of a regular neighborhood of $D$. Now $g_{1} \equiv g_{2}$ outside a regular neighborhood of $D_{\alpha}$, and each loop $l$ in $S^{1} \times S^{1}$ is homotopic relative to the base point to a loop $l^{\prime}$ in $S^{1} \times S^{1}$ such that $l^{\prime} \cap D_{\alpha}=\varnothing$. It follows that $g_{2 .}: \pi_{1}\left(S^{1} \times S^{1}\right) \rightarrow \pi_{1}(N)$ is an isomorphism. Continuing in this fashion we see that it is possible to construct a map $g_{r}: S^{1} \times S^{1} \rightarrow N$ such that $g_{r_{*}}: \pi_{1}\left(S^{1} \times S^{1}\right) \rightarrow \pi_{1}(N)$ is an isomorphism, $g_{r}\left(S^{1} \times S^{1}\right) \subset K_{1}$, and $D \cap g_{r}\left(S^{1} \times S^{1}\right)=\varnothing$. Then we set $K_{2}=\operatorname{cl}\left(K_{1}-\mathscr{U}(D, N)\right)$.

If bd $K_{2}$ is compressible in $N$, we repeat the above process to construct $K_{3}$. Eventually we arrive at a map $g_{s}: S^{1} \times S^{1} \rightarrow N$ and a compact manifold $K_{t} \subset N$ such that $g_{s_{*}}: \pi_{1}\left(S^{1} \times S^{1}\right) \rightarrow \pi_{1}(N)$ is an isomorphism, $g_{s}\left(S^{1} \times S^{1}\right) \subset K_{t}$, and bd $K_{t}$ is incompressible in $N$.

Define $g_{s}^{\prime}: S^{1} \times S^{1} \rightarrow K_{t}$ by $g_{s}^{\prime}(x)=g_{s}(x)$. We have the following commutative diagram where $i_{*}$ is induced by inclusion.

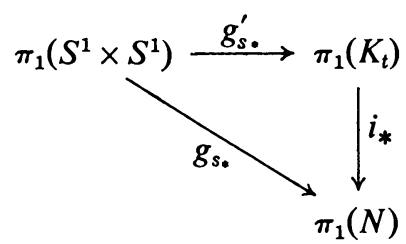

Now $g_{s_{*}}^{\prime}$ and $i_{*}$ are injections and $g_{s_{*}}$ is an isomorphism. Hence $g_{s_{*}}^{\prime}$ and $i_{*}$ are also isomorphisms. Thus $K_{t}$ is a compact 3-manifold with $\pi_{1}\left(K_{t}\right)=Z \oplus Z$. It then follows from a theorem of Heil [4] that $K_{t}$ is equivalent to an $I$ bundle over $S^{1} \times S^{1}$. Since $N$ is orientable we have that in fact $K_{t}$ is equivalent to $S^{1} \times S^{1} \times I$. Let $f: S^{1} \times S^{1}$ 
$\rightarrow K_{t}$ be an embedding such that $f_{*}: \pi_{1}\left(S^{1} \times S^{1}\right) \rightarrow \pi_{1}\left(K_{t}\right)$ is an isomorphism. Then $f$ is the map required by Lemma $\mathrm{A}$.

Let $f\left(S^{1} \times S^{1}\right)=F$. Since $i_{*}: \pi_{1}(F) \rightarrow \pi_{1}(N)$ is an isomorphism $F$ must separate $N$.

LEMMA B. Let $\tau$ be a covering transformation of $(N, p)$ such that $F \cap \tau(F)=\varnothing$. Then $F$ and $\tau(F)$ cobound a manifold $K_{\tau}$ such that $K_{\tau}$ is homeomorphic to $F \times[0,1] \# R$ where $R$ is a possibly noncompact, simply connected 3-manifold.

Proof. Since $F$ and $\tau(F)$ both separate $N, F$ and $\tau(F)$ cobound a manifold $K_{\tau}$. Since $F$ and $\tau(F)$ are incompressible in $N$, we have that $j_{*}: \pi_{1}\left(K_{\tau}\right) \rightarrow \pi_{1}(N)$ is a monomorphism. We have the following commutative diagram of maps induced by inclusion.

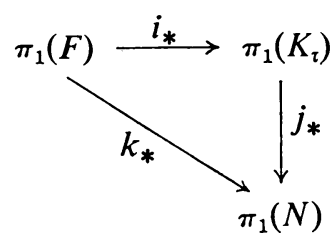

Since each map is a monomorphism and $k_{*}$ is an isomorphism, we have that $i_{*}$ is also an isomorphism. Thus each loop in $\tau(F)$ is freely homotopic in $K_{\tau}$ to a loop in $F$. Then proceeding closely along the lines of Lemma 5.1 of [17] we are able to construct a pair of annuli $A, B$ such that bd $A=a_{1} \cup a_{2}$, bd $B=b_{1} \cup b_{2}$ with the following properties:

(1) $A$ meets $B$ transversely in a single arc $k$ with endpoints $k_{1}, k_{2}$,

(2) $k_{1}=a_{1} \cap b_{1}, k_{2}=a_{2} \cap b_{2}$,

(3) $a_{1}, b_{1}$ is a pair of nontrivial simple closed curves in $\tau(F)$ that meet in a single transverse intersection point $k_{1}$,

(4) $a_{2}, b_{2}$ is a pair of nontrivial simple closed curves in $F$ that meet in a single transverse intersection point $k_{2}$.

Let $X$ denote a regular neighborhood of $F \cup \tau(F) \cup A \cup B$ in $K_{\tau}$. We note that bd $X$ is a 2-sphere in $K_{\tau}$. Since $\pi_{1}\left(K_{\tau}\right)$ is not a nontrivial free product, we must have that bd $X$ bounds a simply connected manifold $R^{*}$ in $K_{\tau}$. Lemma B follows.

LEMMA C. $C$, the group of covering transformations of $(N, p)$, contains an element of infinite order.

Proof. Since $C$ is an infinite group, there is a covering transformation $\tau$ in $C$ such that $F \cap \tau(F)=\varnothing$. Let $F$ separate $N$ into components $N_{1}$ and $N_{2}$. With no loss of generality we assume that $\tau(F) \subset N_{1}$. We have either $\tau\left(N_{1}\right)=\mathrm{cl}\left(N_{1}-K_{\tau}\right)$ or $\tau\left(N_{2}\right)=\mathrm{cl}\left(N_{1}-K_{\tau}\right)$. If $\tau\left(N_{1}\right)=\mathrm{cl}\left(N_{1}-K_{\tau}\right)$, then $\cdots \varsubsetneqq \tau^{2}\left(N_{1}\right) \varsubsetneqq \tau\left(N_{1}\right) \varsubsetneqq N_{1}$. Hence $\tau$ would be of infinite order. Thus we assume that $\tau\left(N_{1}\right)=N_{2} \cup K_{\tau}$, and $\tau\left(N_{2}\right)$ $=\mathrm{cl}\left(N_{1}-K_{\tau}\right)$. By Lemma B we can write $K_{\tau}=K \# R$ where $K$ is compact and $R$ is simply connected. Since for each $\sigma \in C, \sigma(F)$ is incompressible in $N$, it is not possible that $\sigma(F) \subset R$ for any $\sigma \in C$. Thus if $\sigma \in C$, then $\sigma(F) \cap K_{\imath} \neq \varnothing$ if and only if $\sigma(F) \cap K \neq \varnothing$. Since $K$ is compact we have that there are only finitely many 
covering transformations $\sigma$ such that $\sigma(F) \cap K_{\tau} \neq \varnothing$. Thus we are able to find some $\lambda \in C$ such that $\lambda(F) \cap K_{\tau}=\varnothing$. If $\lambda(F) \subset N_{2}$ we put $\lambda_{1}=\tau \lambda$; otherwise, $\lambda_{1}=\lambda$. Thus we have $\lambda_{1} \in C$ such that $\lambda_{1}(F) \subset N_{1}-K_{\tau}$. We have either $\lambda_{1}\left(N_{1}\right)=\operatorname{cl}\left(N_{1}-K_{\lambda_{1}}\right)$ or $\lambda_{1}\left(N_{2}\right)=\mathrm{cl}\left(N_{1}-K_{\lambda_{1}}\right)$. If $\lambda_{1}\left(N_{1}\right)=\mathrm{cl}\left(N_{1}-K_{\lambda_{1}}\right)$, then $\cdots \varsubsetneqq \lambda_{1}^{2}\left(N_{1}\right) \varsubsetneqq \lambda_{1}\left(N_{1}\right) \varsubsetneqq N_{1}$. Hence $\lambda_{1}$ is of infinite order. We are left with the case that $\lambda_{1}\left(N_{2}\right)=\operatorname{cl}\left(N_{1}-K_{\lambda_{1}}\right)$ $\varsubsetneqq \operatorname{cl}\left(N_{1}-K_{\tau}\right)=\tau\left(N_{2}\right)$. Then $\cdots \varsubsetneqq\left(\tau^{-1} \lambda_{1}\right)^{2}\left(N_{2}\right) \varsubsetneqq\left(\tau^{-1} \lambda_{1}\right)\left(N_{2}\right) \varsubsetneqq N_{2}$. Hence $\tau^{-1} \lambda_{1}$ is of infinite order. This completes the proof of Lemma $\mathrm{C}$.

The supposition that $\pi_{1}(M)$ is not of local rank one has now led us to the following situation. $H$ is a subgroup of $\pi_{1}(M)$, and $H$ is isomorphic with $Z \oplus Z$. Furthermore, since $\pi_{1}(M) / H$ is isomorphic with the group of covering transformations of $(N, p)$, we have that $\pi_{1}(M) / H$ contains an element of infinite order. It follows that $\pi_{1}(M)$ contains a subgroup $K$ isomorphic with $Z \oplus Z \oplus Z$. We note that $Z \oplus Z \oplus Z$ is the fundamental group of the closed, aspherical, 3-manifold $S^{1} \times S^{1} \times S^{1}$. Then Theorem 8.8 of [1] allows us to write $M$ as a connected sum, $M=Q \# R$ where $K$ is of finite index in $\pi_{1}(R)$. By van Kampen's theorem, $\pi_{1}(M)$ $\approx \pi_{1}(Q) * \pi_{1}(R)$. But $\pi_{1}(M)$ is not a nontrivial free product. It follows that $\pi_{1}(M) \approx \pi_{1}(R)$ contrary to the assumption that $\pi_{1}(M)$ is not finitely generated. Thus we have in fact that $\pi_{1}(M)$ is a torsion free, abelian group of local rank one. It follows that $\pi_{1}(M)$ is a subgroup of the additive group of rationals.

We do not know if a nonfinitely generated abelian group can occur as a subgroup of the fundamental group of a compact 3-manifold. However each subgroup of the additive group of rationals is the fundamental group of some 3-manifold. In order to show this, it clearly suffices to construct a 3-manifold with fundamental group the additive group of rationals. The example that we shall provide seems to be well known, but we include it for the sake of completeness. The example is a noncompact 3-manifold $M$ which is the complement of a solenoid in $S^{3}$. We construct $M$ as follows.

Let $k_{1}$ be an unknotted simple closed curve in $S^{3}$, and let $T_{1}^{1}$ denote a regular neighborhood of $k_{1}$. Let $T_{1}^{2}=\operatorname{cl}\left(S^{3}-T_{1}^{1}\right)$. Let $x_{1}$ denote the generator of $\pi_{1}\left(T_{1}^{1}\right)$ and $y_{1}$ denote the generator of $\pi_{1}\left(T_{1}^{2}\right)$. Beginning with $T_{1}^{1}$ and $T_{1}^{2}$ we construct two sequences of solid tori $T_{1}^{2} \subset T_{2}^{2} \subset \cdots, T_{1}^{1} \supset T_{2}^{1} \supset \cdots$ where $T_{n+1}^{1}$ and $T_{n+1}^{2}$ are constructed as follows. Let $k_{n+1}$ be a simple closed curve in the interior of $T_{n}^{1}$ such that $k_{n+1}$ is unknotted in $S^{3}$, and $k_{n+1}$ is homotopic in $T_{n}^{1}$ to $x_{n}^{n+1}$ where $x_{n}$ is the generator of $\pi_{1}\left(T_{n}^{1}\right)$. Let $T_{n+1}^{1}$ denote a regular neighborhood of $k_{n+1}$ in $T_{n}^{1}$, and put $T_{n+1}^{2}=\operatorname{cl}\left(S^{3}-T_{n+1}^{1}\right)$. Let $x_{n+1}$ be the generator of $\pi_{1}\left(T_{n+1}^{1}\right)$, and let $y_{n+1}$ be the generator of $\pi_{1}\left(T_{n+1}^{2}\right)$.

Set $M=\bigcup_{n=1}^{\infty} T_{n}^{2}$. Using van Kampen's theorem we obtain a presentation for $\pi_{1}(M)$ as a direct limit of infinite cyclic groups. $\pi_{1}(M)$ is presented by

$$
\left(y_{1}, y_{2}, y_{3}, \ldots \mid y_{1}=y_{2}^{2}, y_{2}=y_{3}^{3}, \ldots\right) \text {. }
$$

Then $\pi_{1}(M)$ is isomorphic with the additive group of rationals under a map which takes $y_{n}$ onto $1 / n$ !. 


\section{REFERENCES}

1. D. B. A. Epstein, Projective planes in 3-manifolds, Proc. London Math. Soc. (3) 11 (1961) 469-484. MR 27 \#2968.

2. R. J. Gregorac, On residually finite generalized free products, Proc. Amer. Math. Soc. 24 (1970), 553-555. MR 41 \#5498.

3. M. Hall, Jr., The theory of groups, Macmillan, New York, 1959. MR 21 \#1996.

4. W. Heil, On $P^{2}$-irreducible 3-manifolds, Bull. Amer. Math. Soc. 75 (1969), 772-775. MR 40 \#4958.

5. W. Jaco, Finitely presented subgroups of three-manifold groups, Invent. Math. 13 (1971), 335-346.

6. I. Kaplansky, Infinite abelian groups, Univ. of Michigan Press, Ann Arbor, Mich., 1954. MR 16, 444.

7. H. Kneser, Geschlossen Flachen in dreidimensionalem Mannigfaltigheiten, Jber. Deutsch. Math.-Verein. 38 (1929), 248-260.

8. S. Mac Lane, Homology, Die Grundlehren der math. Wissenschaften, Band 114, Academic Press, New York; Springer-Verlag, Berlin, 1963. Theorem 7.1, p. 343. MR 28 \#122.

9. W. Magnus, A. Karrass and D. Solitar, Combinatorial group theory: Presentations of groups in terms of generators and relations, Pure and Appl. Math., vol. 13, Interscience, New York, 1966. MR 34 \#7617.

10. W. Massey, Algebraic topology: An introduction, Harcourt, Brace and World, New York, 1967, Chap. 4. MR 35 \#2271.

11. J. W. Milnor, Groups which act on $S^{n}$ without fixed points, Amer. J. Math. 79 (1957), 623-630. MR 19, 761.

12. J. Nielsen, Untersuchungen zur Topologie der geschlossenen zweisentigen Flachen, Acta. Math. 50 (1927), Satz 11, 266.

13. H. Seifert, Topologie dreidimensionalen gefaster Raume, Acta. Math. 60 (1933), 147-238.

14. J. Stallings, On the loop theorem, Ann. of Math. (2) 72 (1960), 12-19. MR 22 \#12526.

15. - On fibering certain 3-manifolds, Topology of 3-Manifolds and Related Topics (Proc. The Univ. of Georgia Inst., 1961), Prentice-Hall, Englewood Cliffs, N. J., 1962, pp. 95-100. MR 28 \#1600.

16. C. Thomas, Nilpotent groups and compact 3-manifolds, Proc. Cambridge Philos. Soc. 64 (1968), 303-306. MR 38 \#1681.

17. F. Waldhausen, On irreducible 3-manifolds which are sufficiently large, Ann. of Math. (2) 87 (1968), 56-88. MR 36 \#7146.

18. - Gruppen mit Zentrum und 3-dimensionale Mannigfaltigkeiten, Topology 6 (1967), 505-517. MR 38 \#5223.

Department of Mathematics, Rice University, Houston, Texas 77001

Department of Mathematics, University of Wisconsin, Madison, Wisconsin 53706

Current address (Benny Evans): Institute for Advanced Study, Princeton, New Jersey 08540

Current address (Louise Moser): Department of Mathematics, California State College at Hayward, Hayward, California 94542 